\title{
BENDING AND BUCKLING OF A METAL SEVEN-LAYER BEAM WITH A LENGTHWISE CORRUGATED MAIN CORE - COMPARATIVE ANALYSIS WITH THE SANDWICH BEAM
}

\author{
Ewa MagnuCKA-BlandZi \\ Poznan University of Technology, Institute of Mathematics, Poznań, Poland \\ e-mail: ewa.magnucka-blandzi@put.poznan.pl \\ MARCIN RODAK \\ Poznan University of Technology, Institute of Applied Mechanics, Poznań, Poland \\ e-mail:marcin.rodak@put.poznan.pl
}

\begin{abstract}
The subject of analytical investigations is a metal seven-layer beam, a plate band with a lengthwise trapezoidal corrugated main core and two crosswise trapezoidal corrugated cores of faces. The hypothesis of deformation of normal to the middle surface of the beam after bending is formulated. Equations of equilibrium are derived based on the theorem of minimum total potential energy. The equations are analytically solved. Three point bending and buckling for axially compression of the simply supported beam are theoretically studied. The deflection and the critical axial force are determined for different values of the trapezoidal corrugation pitch of the main core. Moreover, an adequate model of the sandwich beam with steel foam core is formulated. The deflection and the critical axial force are determined for this sandwich beam. The results studied of the seven layer beam and the adequate sandwich beam are compared and presented in tables and figures.
\end{abstract}

Keywords: layered plate-band, trapezoidal corrugated cores, deflection, critical load

\section{Introduction}

The primary scientific description referring to the analysis and design of sandwich structures is the monograph by Allen (1969). A review of problems related to modelling and calculations of sandwich structures was presented by Noor et al. (1996), Vinson (2001) and Carrera and Brischetto (2009). A developed and analytical model of corrugated composite cores was described by Kazemahvazi and Zenkert (2009). The quasi-isotropic bending response of sandwich plates with bi-directionally corrugated cores was presented by Seong et al. (2010). The mathematical modelling of a rectangular sandwich plate under in plane compression is described by MagnuckaBlandzi (2011). The theoretical study of transverse shear modulus of elasticity for thin-walled corrugated cores of sandwich beams was presented by Magnucka-Blandzi and Magnucki (2014) and Lewinski et al. (2015). The problem of an equivalent plate model for corrugated-core sandwich panels was presented by Cheon and Kim (2015).

The subject of the paper is the metal seven-layer beam - a plate band. The beam is composed of a lengthwise trapezoidal corrugated main core, two inner flat sheets, two crosswise trapezoidal corrugated cores of the faces and two outer flat sheets. 


\section{Theoretical model of the seven-layer beam with the lengthwise corrugated main core}

The seven-layer simply supported beam of length $L$, width $b$, thicknesses of the main core $t_{c 1}$, facing cores $t_{c 2}$ and flat sheets $t_{s}$ is shown in Fig. 1.

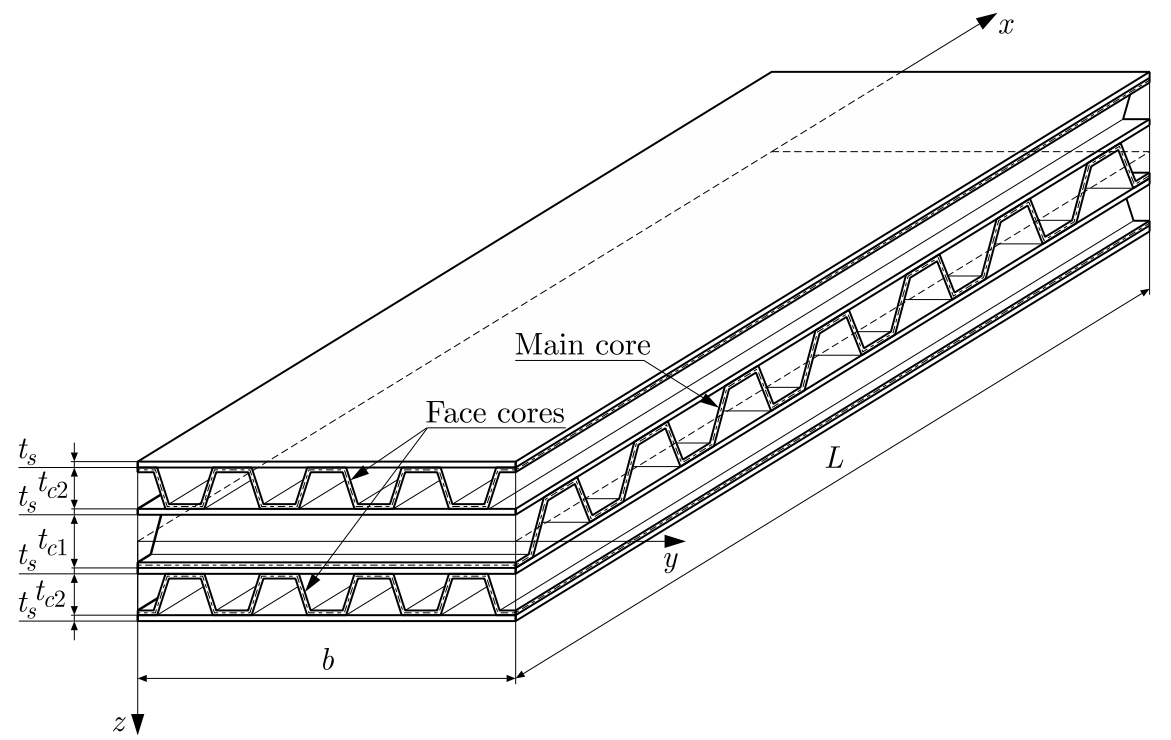

Fig. 1. Scheme of the seven-layer beam with the lengthwise corrugated main core

The directions of corrugations of the main core and the face cores are orthogonal. Trapezoidal corrugations of the main core and facing cores are shown in Fig. 2. The index $i=1$ refers to the main core, while the index $i=2$ refers to the face cores. Total depth of the cores is $t_{c i}$ and length of one pitch of the corrugation is $b_{0 i}$.

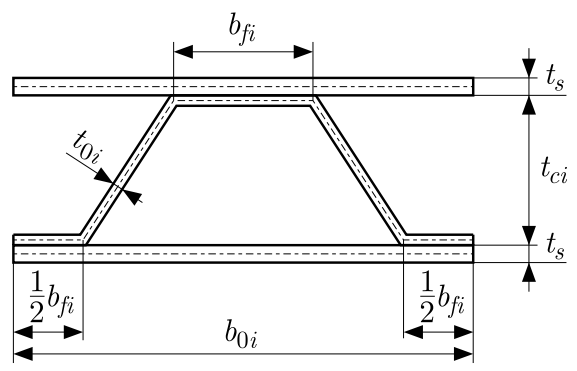

Fig. 2. Scheme of trapezoidal corrugations of the main core $(i=1)$ or face cores $(i=2)$

Taking into account the layered structures of the beam, the hypothesis of the broken line (Fig. 3) is assumed. The plane cross-section before bending does not remain plane and normal after bending. The hypothesis for multi-layer structures was described in details by Carrera (2003), Magnucka-Blandzi (2012) and Magnucki et al. (2016).

The displacements with consideration of this hypothesis are as follows:

- the upper sandwich facing for $-\left(0.5+2 x_{1}+x_{2}\right) \leqslant \zeta \leqslant-0.5$

$$
u(x, y, z)=-t_{c 1}\left[\zeta \frac{d w}{d x}+\psi(x)\right]
$$

- the main corrugated core for $-0.5 \leqslant \zeta \leqslant 0.5$

$$
u(x, z)=-t_{c 1} \zeta\left[\frac{d w}{d x}-2 \psi(x)\right]
$$




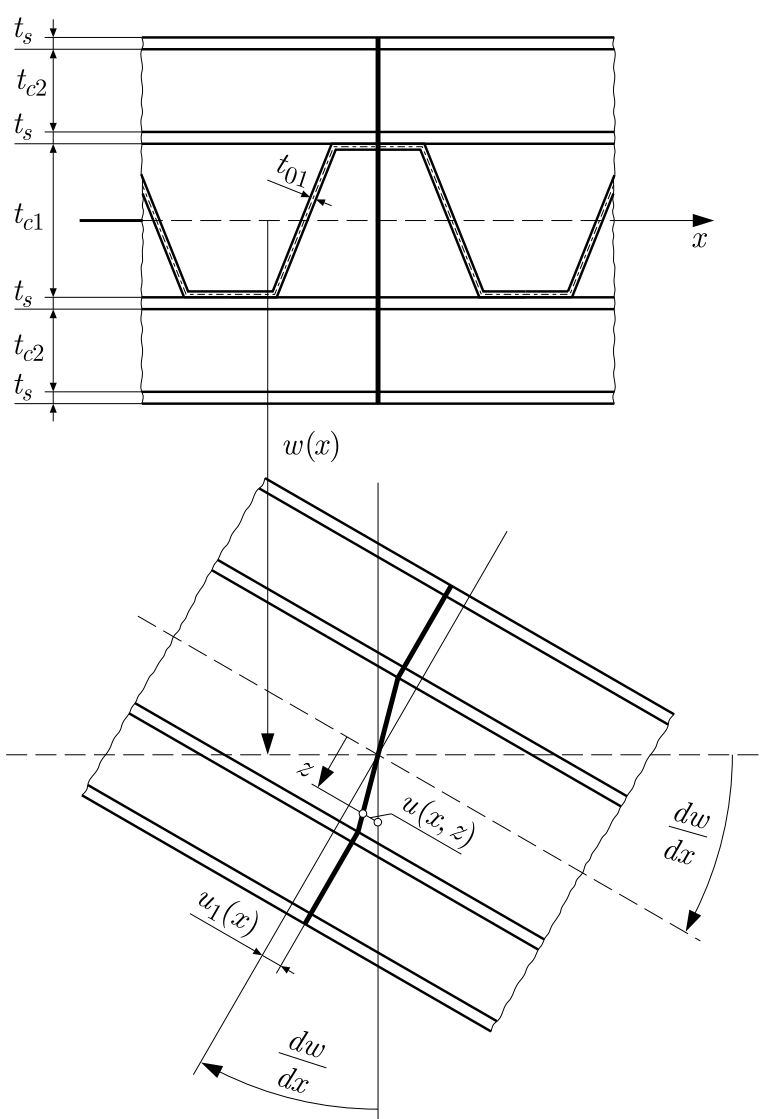

Fig. 3. Scheme of the hypothesis of the seven-layer beam

- the lower sandwich facing for $0.5 \leqslant \zeta \leqslant 0.5+2 x_{1}+x_{2}$

$$
u(x, y, z)=-t_{c 1}\left[\zeta \frac{d w}{d x}-\psi(x)\right]
$$

where $x_{1}=t_{s} / t_{c 1}, x_{2}=t_{c 2} / t_{c 1}$ are dimensionless parameters, $\zeta=z / t_{c 1}$ - dimensionless coordinate, $\psi(x)=u_{1}(x) / t_{c 1}$ - dimensionless functions of displacements, $u_{1}(x)$ - displacement in the $x$ direction and $w(x)$ - deflection (Fig. 3).

Thus, linear relations for the strains are as follows:

- the main corrugated core

$$
\varepsilon_{x}=-t_{c 1} \zeta\left(\frac{d^{2} w}{d x^{2}}-2 \frac{d \psi}{d x}\right) \quad \gamma_{x z}=2 \psi(x)
$$

- the upper/lower sandwich facings

$$
\varepsilon_{x}=-t_{c 1}\left(\zeta \frac{d^{2} w}{d x^{2}} \pm \frac{d \psi}{d x}\right) \quad \gamma_{x z}=0
$$

The sign "+" refers to the upper facing $(u)$, and the sign "_" refers to the lower facing $(l)$.

Strains (2.4) and (2.5) and Hook's law make a basis for the formulation of elastic strain energy of the seven-layer beam.

\section{The equations of equilibrium of the seven-layer beam}

The elastic strain energy of the beam is a sum of the energy of particular layers

$$
U_{\varepsilon}^{(\text {beam })}=U_{\varepsilon}^{(c-1)}+U_{\varepsilon}^{(s-i)}+U_{\varepsilon}^{(c-2)}+U_{\varepsilon}^{(s-o)}
$$


The addends are as follows:

$>$ energy of the main corrugated core

$$
U_{\varepsilon}^{(c-1)}=\frac{1}{2} E_{s} b t_{c 1} \int_{0}^{L} \int_{-\frac{1}{2}}^{\frac{1}{2}}\left[\widetilde{E}_{x}^{(c-1)} \varepsilon_{x}^{2}+\widetilde{G}_{x z}^{(c-1)} \gamma_{x z}^{2}\right] d \zeta d x
$$

where $E_{s}$ is Young's modulus, dimensionless longitudinal elastic modulus of the main corrugated core is calculated based on the monograph of Ventsel and Krauthammer (2001)

$$
\widetilde{E}_{x}^{(c-1)}=\frac{x_{b 1}}{2\left(x_{f 1} x_{b 1}+\widetilde{s}_{a 1}\right)} x_{01}^{3}
$$

dimensionless shear elastic modulus of the main trapezoidal corrugated core based on the paper of Lewinski et al. (2015)

$$
\widetilde{G}_{x z}^{(c-1)}=\frac{1-x_{01}}{4\left(1-\nu^{2}\right) x_{b 1} f_{u}}\left(\frac{x_{01}}{\widetilde{s}_{a 1}}\right)^{3}
$$

and dimensionless parameters

$$
\begin{aligned}
& x_{01}=\frac{t_{01}}{t_{c 1}} \quad x_{f 1}=\frac{b_{f 1}}{b_{01}} \quad x_{b 1}=\frac{b_{01}}{t_{c 1}} \\
& \widetilde{s}_{a 1}=\sqrt{\left(1-x_{01}\right)^{2}+x_{b 1}^{2}\left(\frac{1}{2}-x_{f 1}\right)^{2}}
\end{aligned}
$$

Substituting expressions (2.4) for strains into expression (3.2) and after integration, the elastic energy of the main corrugated core is obtained in the following form

$$
U_{\varepsilon}^{(c-1)}=E_{s} b t_{c 1}^{3} \int_{0}^{L}\left\{\frac{1}{24} \widetilde{E}_{x}^{(c-1)}\left[\left(\frac{d^{2} w}{d x^{2}}\right)^{2}-4 \frac{d^{2} w}{d x^{2}} \frac{d \psi}{d x}+4\left(\frac{d \psi}{d x}\right)^{2}\right]+2 \widetilde{G}_{x z}^{(c-1)}\left(\frac{\psi(x)}{t_{c 1}}\right)^{2}\right\} d x
$$

$>$ energy of the inner sheets

$$
U_{\varepsilon}^{(s-i)}=\frac{1}{2} E_{s} b t_{c 1} \int_{0}^{L}\left\{\int_{-\left(\frac{1}{2}+x_{1}\right)}^{-\frac{1}{2}} \varepsilon_{x, u p}^{2} d \zeta+\int_{\frac{1}{2}}^{\frac{1}{2}+x_{1}} \varepsilon_{x, l o w}^{2} d \zeta\right\} d x
$$

Substitution of expressions (2.5) for the strains with regard to the upper/lower facings and after integration provides

$$
U_{\varepsilon}^{(s-i)}=E_{s} b t_{c 1}^{3} \int_{0}^{L}\left[\frac{1}{12} x_{1}\left(3+6 x_{1}+4 x_{1}^{2}\right)\left(\frac{d^{2} w}{d x^{2}}\right)^{2}-x_{1}\left(1+x_{1}\right) \frac{d^{2} w}{d x^{2}} \frac{d \psi}{d x}+x_{1}\left(\frac{d \psi}{d x}\right)^{2}\right] d x
$$

energy of the corrugated cores of the facings

$$
U_{\varepsilon}^{(c-2)}=\frac{1}{2} E_{s} \frac{b}{b_{02}} \int_{0}^{L}\left[\int_{A_{T R}^{(c-2)}} \varepsilon_{x, u p}^{2} d A_{T R}^{(c-2)}+\int_{A_{T R}^{(c-2)}} \varepsilon_{x, l o w}^{2} d A_{T R}^{(c-2)}\right]
$$


where the area of the trapezoid

$$
A_{T R}^{(c-2)}=2 t_{c 2}^{2} x_{02}\left(x_{f 2} x_{b 2}+\widetilde{s}_{a 2}\right)
$$

and dimensionless parameters

$$
\begin{aligned}
& x_{02}=\frac{t_{02}}{t_{c 2}} \quad x_{f 2}=\frac{b_{f 2}}{b_{02}} \quad x_{b 2}=\frac{b_{02}}{t_{c 2}} \\
& \widetilde{s}_{a 2}=\sqrt{\left(1-x_{02}\right)^{2}+x_{b 2}^{2}\left(\frac{1}{2}-x_{f 2}\right)^{2}}
\end{aligned}
$$

Substituting expressions (2.5) for strains into expression (3.9) and after integration, the elastic energy of the corrugated cores of facings is obtained in the following form

$$
U_{\varepsilon}^{(c-2)}=E_{s} b t_{c 1}^{3} \frac{x_{2} x_{02}}{x_{b 2}} \int_{0}^{L}\left[C_{w w}^{(c-2)}\left(\frac{d^{2} w}{d x^{2}}\right)^{2}-C_{w \psi}^{(c-2)} \frac{d^{2} w}{d x^{2}} \frac{d \psi}{d x}+C_{\psi \psi}^{(c-2)}\left(\frac{d \psi}{d x}\right)^{2}\right] d x
$$

where dimensionless parameters are as follows

$$
\begin{aligned}
& C_{w w}^{(c-2)}=\frac{1}{2}\left[\frac{1}{3} x_{2}^{2}\left(1-x_{02}\right)^{2}\left(3 x_{f 2} x_{b 2}+\widetilde{s}_{a 2}\right)+\left(1+2 x_{1}+x_{2}\right)^{2}\left(x_{f 2} x_{b 2}+\widetilde{s}_{a 2}\right)\right] \\
& C_{w \psi}^{(c-2)}=2\left(1+2 x_{1}+x_{2}\right)\left(x_{f 2} x_{b 2}+\widetilde{s}_{a 2}\right) \quad C_{\psi \psi}^{(c-2)}=2\left(x_{f 2} x_{b 2}+\widetilde{s}_{a 2}\right)
\end{aligned}
$$

$>$ energy of the outer sheets

$$
U_{\varepsilon}^{(s-o)}=\frac{1}{2} E_{s} b t_{c 1} \int_{0}^{L}\left\{\begin{array}{c}
-\left(\frac{1}{2}+x_{1}+x_{2}\right) \\
\int \varepsilon_{x, u p}^{2} d \zeta+\int_{\frac{1}{2}+x_{1}+x_{2}}^{\frac{1}{2}+2 x_{1}+x_{2}} \varepsilon_{x, l o w}^{2} d \zeta
\end{array}\right\} d x
$$

Substitution of expressions (2.5) for the strains with regard to the upper/lower facings and after integration gives

$$
U_{\varepsilon}^{(s-o)}=E_{s} b t_{c 1}^{3} \int_{0}^{L}\left[C_{w w}^{(s-o)}\left(\frac{d^{2} w}{d x^{2}}\right)^{2}-x_{1}\left(1+3 x_{1}+2 x_{2}\right) \frac{d^{2} w}{d x^{2}} \frac{d \psi}{d x}+x_{1}\left(\frac{d \psi}{d x}\right)^{2}\right] d x
$$

where the dimensionless parameter $C_{w w}^{(s-o)}=(1 / 12) x_{1}\left[28 x_{1}^{2}+3\left(1+2 x_{2}\right)\left(1+6 x_{1}+2 x_{2}\right)\right]$.

Therefore, the elastic strain energy of the inner and outer sheets is as follows

$$
U_{\varepsilon}^{(s)}=U_{\varepsilon}^{(s-i)}+U_{\varepsilon}^{(s-o)}=E_{s} b t_{c 1}^{3} \int_{0}^{L}\left[C_{w w}^{(s)}\left(\frac{d^{2} w}{d x^{2}}\right)^{2}-C_{w \psi}^{(s)} \frac{d^{2} w}{d x^{2}} \frac{d \psi}{d x}+2 x_{1}\left(\frac{d \psi}{d x}\right)^{2}\right] d x
$$

where dimensionless parameters

$$
C_{w w}^{(s)}=\frac{1}{6} x_{1}\left[16 x_{1}^{2}+6 x_{1}\left(2+3 x_{2}\right)+3\left(1+2 x_{2}+2 x_{2}^{2}\right)\right] \quad C_{w \psi}^{(s)}=2 x_{1}\left(1+2 x_{1}+x_{2}\right)
$$

Thus, the elastic strain energy of the seven-layer beam (6) is in the following form

$$
\begin{aligned}
& U_{\varepsilon}^{(\text {beam })}=E_{s} b t_{c 1}^{3} \int_{0}^{L}\left[\frac{1}{2} C_{w w}\left(\frac{d^{2} w}{d x^{2}}\right)^{2}-C_{w \psi} \frac{d^{2} w}{d x^{2}} \frac{d \psi}{d x}+\frac{1}{2} C_{\psi \psi}\left(\frac{d \psi}{d x}\right)^{2}\right. \\
&\left.+2 \widetilde{G}_{x z}^{(c-1)}\left(\frac{\psi(x)}{t_{c 1}}\right)^{2}\right] d x
\end{aligned}
$$


where dimensionless parameters

$$
\begin{aligned}
& C_{w w}=\frac{1}{12} \widetilde{E}_{x}^{(c-1)}+2 \frac{x_{2} x_{02}}{x_{b 2}} C_{w w}^{(c-2)}+2 C_{w w}^{(s)} \quad C_{w \psi}=\frac{1}{6} \widetilde{E}_{x}^{(c-1)}+\frac{x_{2} x_{02}}{x_{b 2}} C_{w \psi}^{(c-2)}+C_{w \psi}^{(s)} \\
& C_{w \psi}=\frac{1}{3} \widetilde{E}_{x}^{(c-1)}+2 \frac{x_{2} x_{02}}{x_{b 2}} C_{w \psi}^{(c-2)}+2 C_{w \psi}^{(s)}
\end{aligned}
$$

The work of the load

$$
W=\int_{0}^{L}\left[q w(x)+\frac{1}{2} F_{0}\left(\frac{d w}{d x}\right)^{2}\right] d x
$$

where $q$ is the intensity of the transverse load, $F_{0}$ - axial compressive force of the beam.

The system of the equations of equilibrium - two ordinary differential equations derived based on the theorem of minimum potential energy $\delta\left(U_{\varepsilon}^{(\text {beam })}-W\right)=0$, is in the following form

$$
\begin{aligned}
& C_{w w} \frac{d^{4} w}{d x^{4}}-C_{w \psi} \frac{d^{3} \psi}{d x^{3}}=\frac{1}{E b t_{c 1}^{3}}\left(q-F_{0} \frac{d^{2} w}{d x^{2}}\right) \\
& C_{w \psi} \frac{d^{3} w}{d x^{3}}-C_{\psi \psi} \frac{d^{2} \psi}{d x^{2}}+4 \widetilde{G}_{x z}^{(c-1)} \frac{\psi(x)}{t_{c 1}^{2}}=0
\end{aligned}
$$

The bending moment of the seven-layer beam

$$
M_{b}(x)=\int_{A} z \sigma_{x} d A=-E_{s} b t_{c 1}^{3}\left(C_{w w} \frac{d^{2} w}{d x^{2}}-C_{w \psi} \frac{d \psi}{d x}\right)
$$

Integration is analogical as in the case of the elastic strain energy, from which the following equation is obtained

$$
C_{w w} \frac{d^{2} w}{d x^{2}}-C_{w \psi} \frac{d \psi}{d x}=-\frac{M_{b}(x)}{E_{s} b t_{c 1}^{3}}
$$

Equations (3.18) 1 and (3.20) are equivalent, therefore, bending and buckling analysis of the seven-layer beam is based on the system of two differential equations $(3.18)_{2}$ and (3.20).

\section{Deflection of the seven-layer beam under three-point bending}

Three-point bending of the seven-layer beam of length $L$ is shown in Fig. 4 .

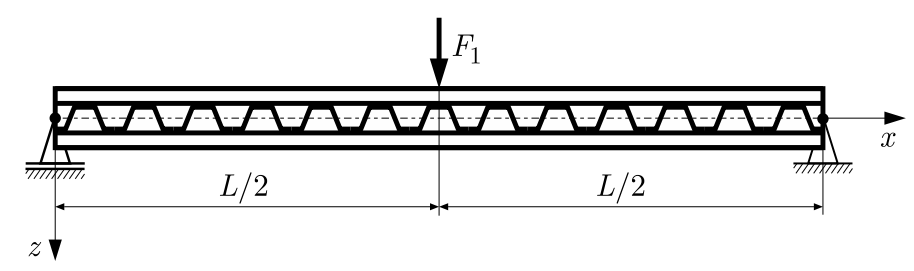

Fig. 4. Scheme of the three-point bending of the beam

The system of two differential equations $(3.18)_{2}$ and (3.20) is reduced to one differential equation in the following form

$$
\frac{d^{2} \psi}{d x^{2}}-\left(\frac{k}{t_{c 1}}\right)^{2} \psi(x)=-C_{q} \frac{Q(x)}{E_{s} b t_{c 1}^{3}}
$$


where $Q(x)=d M_{b} / d x$ is the shear force, $k, C_{q}$ - dimensionless parameters

$$
k=2 \sqrt{\frac{C_{w w} \widetilde{G}_{x z}^{(c-1)}}{C_{w w} C_{\psi \psi}-C_{w \psi}^{2}}} \quad C_{q}=\frac{C_{w \psi}}{C_{w w} C_{\psi \psi}-C_{w \psi}^{2}}
$$

The general solution to equation (4.1) is in the form

$$
\psi(x)=C_{1} \sinh \left(k \frac{x}{t_{c 1}}\right)+C_{2} \cosh \left(k \frac{x}{t_{c 1}}\right)+\psi_{p}(x)
$$

where $C_{1}, C_{2}$ are integration constants, $\psi_{p}(x)$ - particular solution.

The shear force in the half beam (Fig. 4 ) is $Q(x)=F_{1} / 2$, for $0 \leqslant x \leqslant L / 2$, then the particular solution

$$
\psi_{p}=\frac{C_{w \psi}}{8 C_{w w} \widetilde{G}_{x z}^{(c-1)}} \frac{F_{1}}{E_{s} b t_{c 1}}
$$

Taking into account the boundary conditions for the half beam $\left.(d \psi / d x)\right|_{x=0}=0$ and $\psi(L / 2)=0$, the integration constants $C_{1}=0$ and $C_{2}=-\cosh ^{-1}\left[k L /\left(2 t_{c 1}\right)\right] \psi_{0}$ are determined, hence, the function of displacement (4.3) is in the following form

$$
\psi(x)=\left(1-\frac{\cosh \frac{k x}{t_{c 1}}}{\cosh \frac{k L}{2 t_{c 1}}}\right) \psi_{p}
$$

Substituting this function, and the bending moment $M_{b}(x)=F_{1} x / 2$, for $0 \leqslant x \leqslant L / 2$ to equation (3.20), one obtains

$$
w(x)=C_{4}+C_{3} x+\frac{C_{w \psi}}{C_{w w}}\left(x-\frac{t_{c 1}}{k} \frac{\sinh \frac{k x}{t_{c 1}}}{\cosh \frac{k L}{2 t_{c 1}}}\right) \psi_{p}-\frac{F_{1}}{12 C_{w w} E_{s} b t_{c 1}^{3}} x^{3}
$$

Taking into account the boundary conditions for the half beam $w(0)=0$ and $\left.(d w / d x)\right|_{x=L / 2}=0$, the integration constants $C_{3}=F_{1} L^{2} /\left(16 C_{w w} E_{s} b t_{c 1}^{3}\right)$ and $C_{4}=0$ are determined. The maximum deflection - the deflection for the middle of the beam is

$$
w_{\text {max }}^{(7-l a y)}=w\left(\frac{L}{2}\right)=\left[1+3\left(1-\frac{2 t_{c 1}}{k L} \tanh \frac{k L}{2 t_{c 1}}\right) \frac{C_{w \psi}^{2}}{C_{w w} \widetilde{G}_{x z}^{(c-1)}}\left(\frac{t_{c 1}}{L}\right)^{2}\right] \frac{F_{1}}{48 C_{w w} E_{s} b}\left(\frac{L}{t_{c 1}}\right)^{3}
$$

\section{Critical load of the seven-layer beam subjected to axial compression}

The axial compression of the simply supported seven-layer beam is shown in Fig. 5 .

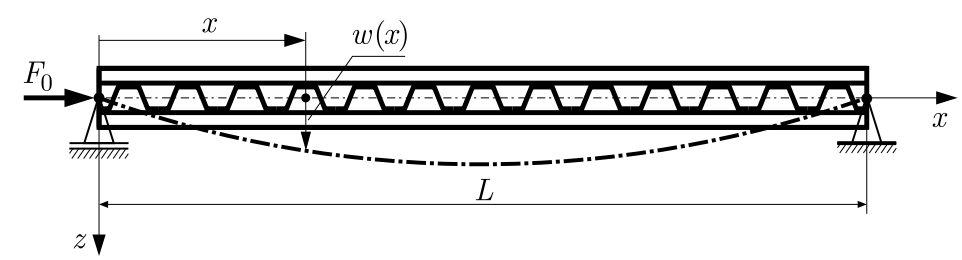

Fig. 5. Scheme of the simply supported seven-layer beam with the axial force $F_{0}$

The system of two differential equations $(3.18)_{2}$ and (3.20) is reduced to one differential equation in the following form

$$
\left(C_{w w} C_{\psi \psi}-C_{w \psi}^{2}\right) \frac{d^{4} w}{d x^{4}}-\frac{4}{t_{c 1}^{2}} \widetilde{G}_{x z}^{(c-1)} C_{w w} \frac{d^{2} w}{d x^{2}}=\left[\frac{4}{t_{c 1}^{2}} \widetilde{G}_{x z}^{(c-1)} M_{b}(x)-C_{\psi \psi} \frac{d^{2} M_{b}}{d x^{2}}\right] \frac{1}{E_{s} b t_{c 1}^{3}}
$$

where the bending moment $M_{b}(x)=F_{0} w(x)$ (Fig. 5). 
Differential equation (5.1) with one unknown function $w(x)$ is approximately solved assuming this function in the form

$$
w(x)=w_{a} \sin \frac{\pi x}{L}
$$

where $w_{a}$ is the parameter of the function, $L$ - length of the beam.

Substituting this function into the equation (5.1) the critical force is obtained

$$
F_{0, C R}^{(7-l a y)}=\left(C_{w w}-\frac{C_{w \psi}^{2}}{\alpha_{1}}\right) \frac{\pi^{2} E_{s} b t_{c 1}^{3}}{L^{2}}
$$

where

$$
\alpha_{1}=C_{\psi \psi}+\left(\frac{2 L}{\pi t_{c 1}}\right)^{2} \widetilde{G}_{x z}^{(c-1)}
$$

\section{Equivalent sandwich beam}

Comparative analysis is carried out for the classical sandwich beam (Fig. 6) equivalent to the seven-layer beam (Fig. 1). This classical sandwich beam consists of two steel faces of thickness $t_{f}=t_{s}$ and the steel foam core of thickness $t_{c}=t_{c 1}+2\left(t_{s}+t_{c 2}\right)$. Its sizes and mass are identical to the seven-layer beam.

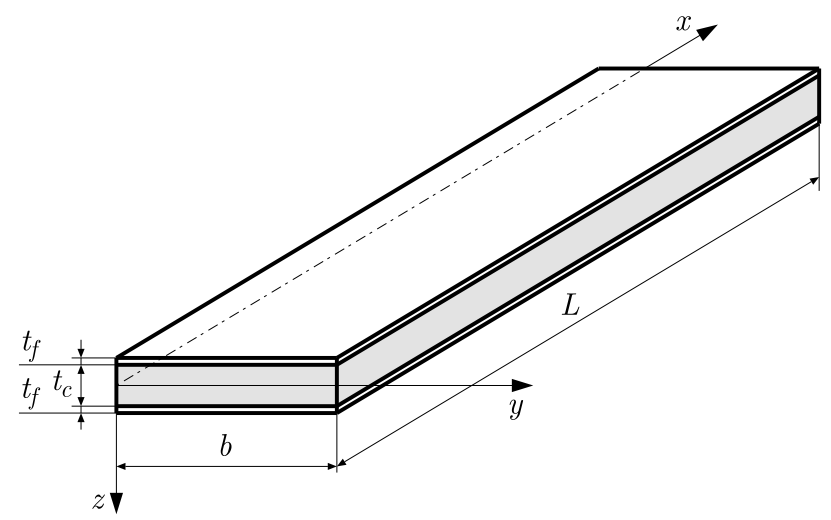

Fig. 6. Scheme of the sandwich (three-layer) beam equivalent to the seven-layer beam

The mass of the metal foam core of this sandwich beam (three-layer beam)

$$
m_{c}^{(3-l a y)}=\left[1+2\left(x_{1}+x_{2}\right)\right] t_{c 1} b L \rho_{c}
$$

where $\rho_{c}$ is the mass density of the metal foam core.

However, mass of the material (steel with mass density $\rho_{s}$ ) located between the two outer sheets of the seven-layer beam (Fig. 1) is a sum of the mass of particular layers

$$
m_{c}^{(7-l a y)}=m_{c}^{(c-1)}+2 m_{c}^{(s-i)}+2 m_{c}^{(c-2)}
$$

where the mass of the main corrugated core

$$
m_{c}^{(c-1)}=\frac{A_{T R}^{(c-1)}}{b_{01}} b L \rho_{s}
$$

Substituting the expression for the area of the trapezoid $A_{T R}^{(c-1)}=2 t_{c 1}^{2} x_{01}\left(x_{f 1} x_{b 1}+\widetilde{s}_{a 1}\right)$ to the above expression with consideration of the dimensionless parameters (3.5) one obtains

$$
m_{c}^{(c-1)}=2 x_{01}\left(x_{f 1}+\frac{\widetilde{s}_{a 1}}{x_{b 1}}\right) t_{c 1} b L \rho_{s}
$$


and

$$
m_{c}^{(s-i)}=t_{s} b L \rho_{s}=x_{1} t_{c 1} b L \rho_{s} \quad m_{c}^{(c-2)}=\frac{A_{T R}^{(c-2)}}{b_{02}} b L \rho_{s}
$$

where $m_{c}^{(s-i)}$ is the mass of the inner sheets, $m_{c}^{(c-2)}$ - mass of the corrugated cores of the facings.

Substituting the expression for the area of trapezoid (3.10) with dimensionless parameters (3.11), one obtains

$$
m_{c}^{(c-2)}=2 x_{2} x_{02}\left(x_{f 2}+\frac{\widetilde{s}_{a 2}}{x_{b 2}}\right) t_{c 1} b L \rho_{s}
$$

Thus, mass (6.2) is in the following form

$$
m_{c}^{(7-l a y)}=2\left[x_{01}\left(x_{f 1}+\frac{\widetilde{s}_{a 1}}{x_{b 1}}\right)+x_{1}+x_{2} x_{02}\left(x_{f 2}+\frac{\widetilde{s}_{a 2}}{x_{b 2}}\right)\right] t_{c 1} b L \rho_{s}
$$

Then, from the equivalence condition $m_{c}^{(3-l a y)}=m_{c}^{(7-l a y)}$ (Eqs. (6.1) and (6.7)) of these two beams, the proportion of mass densities of the metal foam core to steel is obtained

$$
\widetilde{\rho}_{c}=\frac{\rho_{c}}{\rho_{s}}=\left[x_{01}\left(x_{f 1}+\frac{\widetilde{s}_{a 1}}{x_{b 1}}\right)+x_{1}+x_{2} x_{02}\left(x_{f 2}+\frac{\widetilde{s}_{a 2}}{x_{b 2}}\right)\right] \frac{2}{1+2\left(x_{1}+x_{2}\right)}
$$

Taking into account the experimental results related to the mechanical properties of metal foams presented in details by Ashby et al. (2000), Smith et al. (2012) and Szyniszewski et al. (2014), the relationship for Young's moduli and mass densities of the metal foams and the reference material (steel) is as follows

$$
\widetilde{E_{c}}=\frac{E_{c}}{E_{s}}=\frac{3}{4}\left(\frac{\rho_{c}}{\rho_{s}}\right)^{2}
$$

where $E_{c}$ and $E_{s}$ are Young's moduli of the metal foam and the steel.

\section{Bending and buckling of the equivalent sandwich beam}

The hypothesis of deformation of the plane cross-section after bending of the sandwich (three-layer) beam is assumed as the broken line (Fig. 7). The detailed description of this hypothesis and derivation of the equations of equilibrium for the sandwich beam was presented by Magnucka-Blandzi (2012).

The displacements with consideration of this hypothesis are as follows:

- the upper/lower facing for $-\left(0.5+x_{0}\right) \leqslant \zeta \leqslant-0.5$ and $0.5 \leqslant \zeta \leqslant 0.5+x_{0}$

$$
u(x, z)=-t_{c}\left[\zeta \frac{d w}{d x} \pm \psi_{0}(x)\right]
$$

- the metal foam core for $-0.5 \leqslant \zeta \leqslant 0.5$

$$
u(x, z)=-t_{c} \zeta\left[\frac{d w}{d x}-2 \psi_{0}(x)\right]
$$

where $x_{0}=t_{f} / t_{c}$ is the dimensionless parameter, $\zeta=z / t_{c}-$ dimensionless coordinate, $\psi_{0}(x)=u_{f}(x) / t_{c}$ - dimensionless functions of displacements, $u_{f}(x)$ - displacement in the $x$ direction and $w(x)$ - deflection (Fig. 7). 


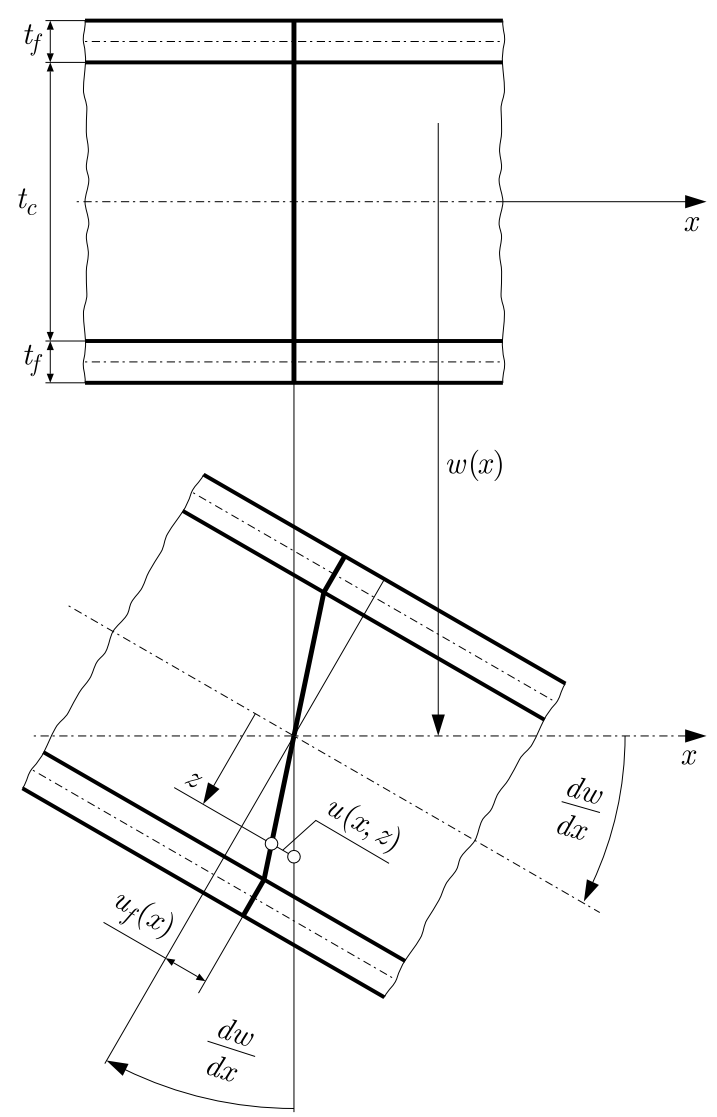

Fig. 7. Scheme of the hypothesis of the sandwich (three-layer) beam

Continuation of the procedure similar to the one applied to the seven-layer beam gives a system of two differential equations of equilibrium for the classical sandwich beam presented by Magnucka (2012) in the following form

$$
B_{w w} \frac{d^{2} w}{d x^{2}}-B_{w \psi} \frac{d \psi_{0}}{d x}=-\frac{M_{b}(x)}{E_{s} b t_{c}^{3}} \quad B_{w \psi} \frac{d^{3} w}{d x^{3}}-B_{\psi \psi} \frac{d^{2} \psi_{0}}{d x^{2}}+4 \widetilde{G}_{c} \frac{\psi_{0}(x)}{t_{c}^{2}}=0
$$

where dimensionless parameters

$$
\begin{aligned}
B_{w w} & =2 C_{2 f}+\frac{1}{12} \widetilde{E}_{c} & B_{w \psi} & =C_{1 f}+\frac{1}{6} \widetilde{E}_{c} \quad B_{\psi \psi}=2 x_{0}+\frac{1}{3} \widetilde{E}_{c} \\
C_{1 f} & =\left(1+x_{0}\right) x_{0} & C_{2 f} & =\frac{1}{12}\left(3+6 x_{0}+4 x_{0}^{2}\right) x_{0}
\end{aligned}
$$

and moduli

$$
\widetilde{E_{c}}=\frac{E_{c}}{E_{s}} \quad \widetilde{G_{c}}=\frac{\widetilde{E}_{c}}{2\left(1+\nu_{c}\right)}
$$

This system of equations is analogical to the one of the seven-layer beam, (3.20) and (3.18) 2 .

Then, the maximum deflection and the critical force of the sandwich equivalent beam are as follows

$$
w_{\text {max }}^{(3-\text { lay })}=w\left(\frac{L}{2}\right)=\left[1+3\left(1-\frac{2 t_{c}}{k_{0} L} \tanh \frac{k_{0} L}{2 t_{c}}\right) \frac{B_{w \psi}^{2}}{B_{w w} \widetilde{G}_{c}}\left(\frac{t_{c}}{L}\right)^{2}\right] \frac{F_{1}}{48 B_{w w} E_{s} b}\left(\frac{L}{t_{c}}\right)^{3}
$$

and

$$
F_{0, C R}^{(3-l a y)}=\left(B_{w w}-\frac{B_{w \psi}^{2}}{\alpha_{0}}\right) \frac{\pi^{2} E_{s} b t_{c}^{3}}{L^{2}}
$$


where

$$
\alpha_{0}=B_{\psi \psi}+\left(\frac{2 L}{\pi t_{c}}\right)^{2} \widetilde{G_{c}}
$$

\section{Illustrative detailed analysis for selected beams}

A detailed analysis for an examplary steel seven-layer beam and the equivalent sandwich beam is carried out for the following test data: $L=1620 \mathrm{~mm}, b=240 \mathrm{~mm}, t_{s}=0.8 \mathrm{~mm}, t_{c 1}=32.0 \mathrm{~mm}$, $t_{01}=0.8 \mathrm{~mm}, b_{f 1}=10.0 \mathrm{~mm}, b_{01}=[32.4,36.0,40.5,45.0] \mathrm{mm}, t_{c 2}=16.0 \mathrm{~mm}, t_{02}=0.8 \mathrm{~mm}$, $b_{f 2}=8.0 \mathrm{~mm}, b_{02}=40.0 \mathrm{~mm}$ and material-steel constants $E_{s}=2 \cdot 10^{5} \mathrm{MPa}, \nu=0.3$, $\rho_{s}=7850 \mathrm{kgm}^{-3}$. Moreover, $t_{f}=t_{s}=0.8 \mathrm{~mm}$ and $t_{c}=t_{c 1}+2\left(t_{s}+t_{c 2}\right)=65.6 \mathrm{~mm}$.

The values of maximum deflections (4.6) and critical forces (5.3) of the seven-layer beam are specified in Table 1. The values of maximum deflections (7.4) and critical forces (7.5) of the sandwich (three-layer) beam are specified in Table 2.

Table 1. Maximum deflections and critical forces of the seven-layer beam

\begin{tabular}{|c|c|c|c|c|}
\cline { 2 - 5 } \multicolumn{1}{c|}{} & \multicolumn{4}{|c|}{$b_{01}[\mathrm{~mm}]$} \\
\cline { 2 - 5 } \multicolumn{1}{c|}{} & 32.4 & 36.0 & 40.5 & 45.0 \\
\hline \hline$w_{\text {max }}^{(7-\text { lay })}[\mathrm{mm}]$ & 3.49 & 3.18 & 2.98 & 2.88 \\
\hline$F_{0, C R}^{(7-\text { lay }}[\mathrm{kN}]$ & 490.1 & 535.8 & 568.5 & 587.3 \\
\hline
\end{tabular}

Table 2. Maximum deflections and critical forces of the sandwich beam

\begin{tabular}{|c|c|c|c|c|}
\cline { 2 - 5 } \multicolumn{1}{c|}{} & \multicolumn{4}{c|}{$b_{01}[\mathrm{~mm}]$} \\
\cline { 2 - 5 } & 32.4 & 36.0 & 40.5 & 45.0 \\
\hline \hline$\widetilde{\rho_{c}}$ Eq. $(6.8)$ & 0.0892374 & 0.0863605 & 0.0835631 & 0.0814007 \\
\hline$\widetilde{E_{c} \text { Eq. }(6.9)}$ & 0.005972 & 0.005594 & 0.005237 & 0.004970 \\
\hline$w_{\text {max }}^{(3-\text { lay })}[\mathrm{mm}]$ & 5.13 & 5.16 & 5.21 & 5.24 \\
\hline$F_{0, C R}^{(3-\text { lay })}[\mathrm{kN}]$ & 328.1 & 325.6 & 323.2 & 321.3 \\
\hline
\end{tabular}

Moreover, the values of maximum deflections and critical forces of the seven-layer beam and the equivalent sandwich beam are presented in Figs. 8 and 9.

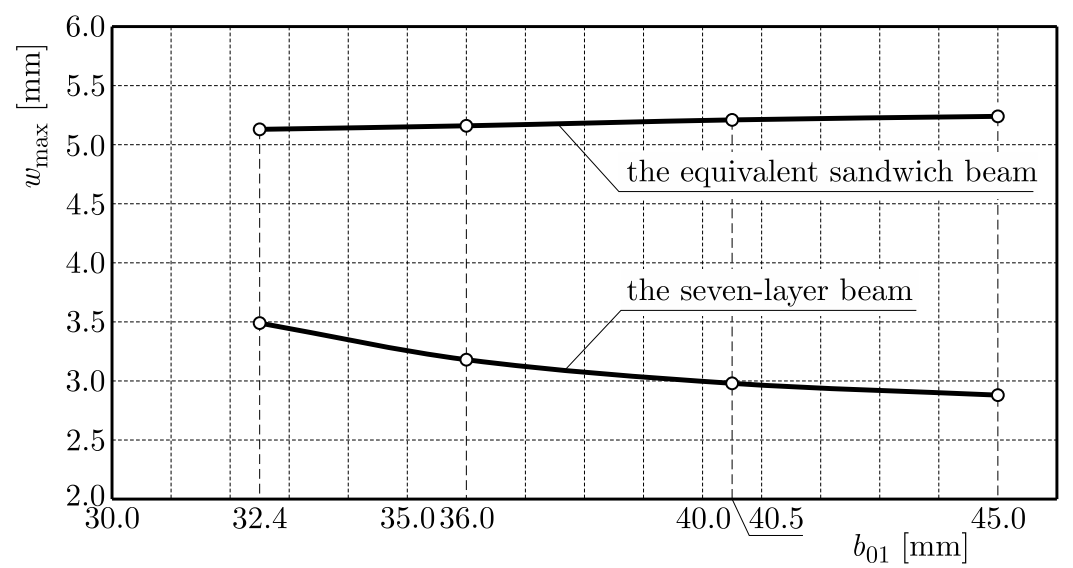

Fig. 8. Maximum deflections of the two beams 


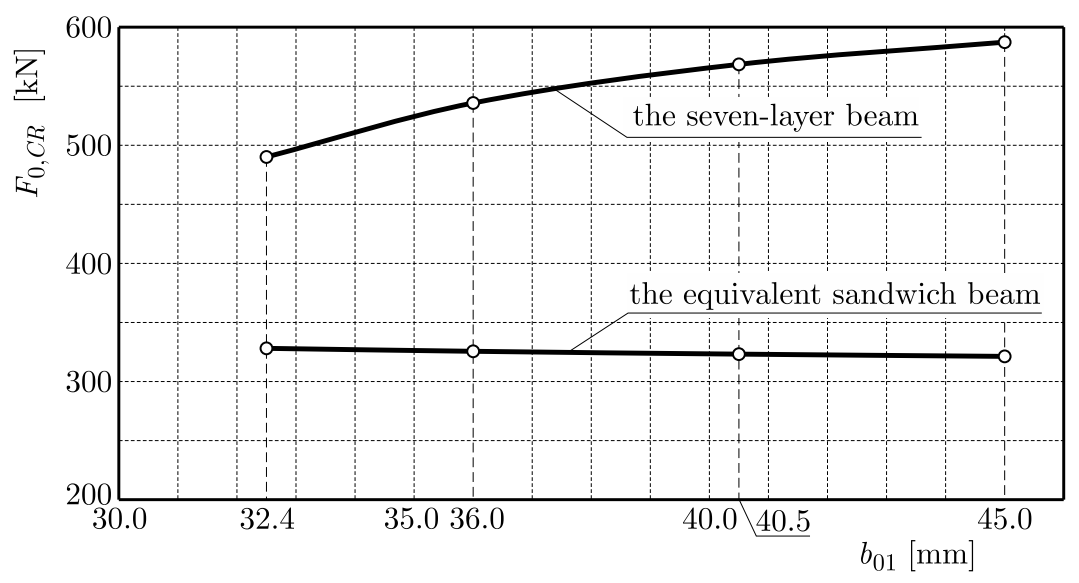

Fig. 9. Critical forces of the two beams

\section{Conclusions}

The analytical modelling of the seven-layer beam with a lengthwise trapezoidal corrugated main core and two crosswise trapezoidal corrugated cores of faces leads to the conclusions:

- hypotheses of the flat cross-sections deformations of these two beams as the broken line are analogous,

- equations of equilibrium of these two beams are similar,

- proportion of the maximum deflections of these two beams for the studied family of the beams is $w_{\max }^{(3-\text { lay })} / w_{\max }^{(7-\text { lay })}=1.47-1.82$,

- proportion of the critical force of these two beams for the studied family of the beams is $F_{0, C R}^{(7-\text { lay })} / F_{0, C R}^{(3-\text { lay })}=1.49-1.83$,

- stiffness of the seven-layer beam is decidedly greater than that of the equivalent classical sandwich (three-layer) beam.

\section{Acknowledgements}

The project was funded by the National Science Centre allocated on the basis of the decision number DEC-2013/09/B/ST8/00170.

\section{References}

1. Allen H.G., 1969, Analysis and Design of Structural Sandwich Panels, Pergamon Press, Oxford, London, Edinburgh, New York, Toronto, Sydney, Paris, Braunschweig

2. Ashby M.F., Evans A., Fleck N.A., Gibson L.J., Hutchinson J.W., Wadley H.N.G., 2000, Metal Foams, A Design Guide, Butterworth-Heinemann, An Imprint of Elsevier

3. Carrera E., 2003, Historical review of Zig-Zag theories for multi-layered plates and shells, Applied Mechanics Reviews, 56, 3, 287-308

4. Carrera E., Brischetto S., 2009, A survey with numerical assessment of classical and refined theories for the analysis of sandwich plates, Applied Mechanics Reviews, 62, 1, 010803

5. Cheon Y.-J., Kim H.-G., 2015, An equivalent plate model for corrugated-core sandwich panels, Journal of Mechanical Science and Technology, 29, 3, 1217-1223

6. Kazemahvazi S., Zenkert D., 2009, Corrugated all-composite sandwich structures. Part 1: Modeling, Composite Science and Technology, 69, 7/8, 913-919 
7. Lewinski J., Magnucka-Blandzi E., Szyc W., 2015, Determination of shear modulus of elasticity for thin-walled trapezoidal corrugated cores of seven-layer sandwich plates, Engineering Transactions, 63, 4, 421-437

8. MagnucKA-Blandzi E., 2011, Mathematical modelling of a rectangular sandwich plate with a metal foam core, Journal of Theoretical and Applied Mechanics, 49, 2, 439-455

9. Magnucka-Blandzi E., 2012, Displacement models of sandwich structures (in Polish), [In:] Strength and Stability Sandwich Beams and Plates with Aluminium Foam Cores, K. Magnucki, W. Szyc (Eds.), Pub. House of Poznan University of Technology, Poznan, 109-120

10. Magnucka-Blandzi E., Magnucki K., 2014, Transverse shear modulus of elasticity for thinwalled corrugated cores of sandwich beams, Theoretical study, Journal of Theoretical and Applied Mechanics, 52, 4, 971-980

11. Magnucka-Blandzi E., Magnucki K., Wittenbeck L., 2015, Mathematical modelling of shearing effect for sandwich beams with sinusoidal corrugated cores, Applied Mathematical Modelling, 39, 2796-2808

12. Magnucki K., Magnucka-Blandzi E., Wittenbeck L., 2016, Elastic bending and buckling of a steel composite beam with corrugated main core and sandwich faces - Theoretical study, Applied Mathematical Modelling, 40, 1276-1286

13. Noor A.K., Burton W.S., Bert C.W., 1996, Computational models for sandwich panels and shells, Applied Mechanics Reviews, 49, 3, 155-199

14. Seong D.Y., Jung C.G., Yang D.Y., Moon K.J., Ahn D.G., 2010, Quasi-isotropic bending responses of metallic sandwich plates with bi-directionally corrugated cores, Materials and Design, 31, 6, 2804-2812

15. Smith B.H., Szyniszewski S., Hajuar J.F., Schafer B.W., Arwade S.R., 2012, Steel foam for structures: A review of applications, manufacturing and material properties, Journal of Constructional Steel Research, 71, 1-10

16. Szyniszewski S., Smith B.H., Hajjar J.F., Schafer B.W., Arwade S.R., 2014, The mechanical properties and modelling of a sintered hollow sphere steel foam, Materials and Design, 54, 1083-1094

17. Ventsel E., Krauthammer T., 2001, Thin Plates and Shells, Theory, Analysis and Applications, Marcel Dekker, New York, Basel

18. Vinson JR., 2001, Sandwich structures, Applied Mechanics Reviews, 54, 3, 201-214 